\title{
Back to the Keller-Osserman Condition for Boundary Blow-up Solutions
}

\section{Serge Dumont*, Louis Dupaigne* \\ Olivier Goubet*}

LAMFA CNRS UMR 6140

Université de Picardie Jules Verne, 33 rue Saint-Leu 80039 Amiens cedex, France

e-mail: serge.dumont@u-picardie.fr

e-mail: louis.dupaigne@u-picardie.fr

e-mail: olivier.goubet@u-picardie.fr

Vicentiu Rădulescu*

Department of Mathematics

University of Craiova, 200585 Craoiva, Romania

e-mail: vicradulescu@yahoo.com

Received 4 December 2006

Communicated by Laurent Véron

\begin{abstract}
This article is concerned with the existence, uniqueness and numerical approximation of boundary blow up solutions for elliptic PDE's $\Delta u=f(u)$, where $f$ satisfies the so-called Keller-Osserman condition. We characterize existence of such solutions for non-monotone $f$. As an example, we construct an infinite family of boundary blow up solutions for the equation $\Delta u=u^{2}(1+\cos u)$ on a ball. We prove uniqueness (on balls) when $f$ is increasing and convex in a neighborhood of infinity and we discuss and perform some numerical computations to approximate such boundary blow-up solutions.
\end{abstract}

2000 AMS Mathematics Subject Classification. 35J60.

Key words. Elliptic equations, boundary blow-up, Keller-Osserman condition

* The authors acknowledge the financial support of PAI Egide Brancusi. 
( In memory of Thomas Lachand-Robert)

\section{Introduction}

\subsection{Setting of the problem}

Let $f$ be a nonnegative function defined on $[0,+\infty)$ such that $f(0)=0$. We assume, for the sake of simplicity, that $f$ is a $C^{1}$ function. Considering $\Omega$ a smooth bounded domain of $\mathbb{R}^{D}, D \geq 1$, we seek $u>0$ a smooth function such that

$$
\left\{\begin{array}{c}
\Delta u=f(u) \text { in } \Omega, \\
u=+\infty \text { on } \partial \Omega,
\end{array}\right.
$$

where the boundary condition is to be understood as :

$$
\lim _{x \rightarrow x_{0}} u(x)=+\infty \quad \forall x_{0} \in \partial \Omega .
$$

To prove existence of such a boundary blow-up solution, it is classically assumed that $f$ is a nondecreasing function with suitable growth rate at infinity, as demonstrated independently by Keller [10] and Osserman [14].

In this article, we study existence, asymptotic behaviour, uniqueness and numerical approximation of solutions of (1.1), when $f$ may exhibit non monotone behaviour.

\subsubsection{Existence results}

Existence of solutions of (1.1) is closely related to the following growth conditions : for $s \in[0,+\infty)$, let $F(s)=\int_{0}^{s} f(t) d t$ and define $\Phi:(0,+\infty) \rightarrow(0,+\infty]$ by

$$
\Phi(\alpha)=\frac{1}{\sqrt{2}} \int_{\alpha}^{\infty} \frac{d s}{\sqrt{F(s)-F(\alpha)}},
$$

where we let by convention $\Phi(\alpha)=+\infty$, whenever the integral is divergent or $F(s)=F(\alpha)$ on a set of positive measure.

Definition 1.1 We say that $f$ satisfies the Keller-Osserman condition whenever

$$
\exists \alpha>0 \quad \Phi(\alpha)<\infty .
$$

We say that $f$ satisfies the Sharpened Keller-Osserman condition whenever

$$
\liminf _{\alpha \rightarrow \infty} \Phi(\alpha)=0 .
$$

Clearly, the Sharpened Keller-Osserman condition implies the classical one. It turns out that both conditions are equivalent (see the Appendix for a proof) :

Proposition 1.2 Assume (1.2) holds for some $\alpha>0$. Then (1.3) holds. 
We point out that in general, $\lim _{\alpha \rightarrow \infty} \Phi(\alpha)$ may not exist : for example if $f(u)=$ $u^{2}(1+\cos u), \lim \sup _{\alpha \rightarrow \infty} \Phi(\alpha)=+\infty$. However, (1.3) still holds. See the Appendix for a proof.

With these definitions in mind, our main result concerning existence reads as follows :

Theorem 1.3 The following statements are equivalent

- $f$ satisfies the Keller-Osserman condition (1.2)

- $f$ satisfies the Sharpened Keller-Osserman condition (1.3)

- There exists a ball $\Omega=B_{R}$ such that (1.1) admits (at least) a positive boundary blow-up solution.

- Given any (smooth bounded) domain $\Omega$, (1.1) admits (at least) a positive boundary blow-up solution.

Theorem 1.3 is a straightforward consequence of Proposition 1.2 and the following two theorems :

Theorem $1.1 f$ satisfies the Keller-Osserman condition if and only if (1.1) admits (at least) a positive boundary blow-up solution on some ball.

Theorem $1.4 f$ satisfies the Sharpened Keller-Osserman condition if and only if (1.1) admits (at least) a positive boundary blow-up solution on any (smooth bounded) domain $\Omega$.

In particular, Theorem 1.4 implies existence of boundary blow-up solutions for functions such as $f(u)=u^{2}(1+\cos u)$.

\subsubsection{Asymptotic behaviour}

The blow-up rate of solutions of (1.1) is determined implicitly by the following theorem :

Theorem 1.5 Assume $\Omega$ satisfies uniform interior and exterior sphere conditions on its boundary. Assume (1.3) holds and let $u$ denote any positive solution of (1.1). Then,

$$
\lim _{x \rightarrow x_{0}} \frac{\int_{u(x)}^{\infty} \frac{d t}{\sqrt{2 F(t)}}}{\delta(x)}=1,
$$

where $\delta(x)=\operatorname{dist}(x, \partial \Omega)$. 


\subsubsection{Uniqueness results}

In view of the maximum principle, it seems natural to only assume that $f$ is nondecreasing in order to obtain uniqueness. To the best of our knowledge, no proof (or counter-example) of such a statement has been given yet. Extra requirements such as the convexity of $f$ or the monotony of $f(u) / u$ are needed in the proofs found in the literature (see e.g. [13]). When the domain is a ball, we relax such assumptions as follows:

Theorem 1.6 Assume that $f$ is nondecreasing and that $f$ is convex in a neighborhood of $+\infty$. Assume also that $f$ satisfies the Keller-Osserman condition (1.2). Then on any ball $B(0, R)$, there exists a unique boundary blow up solution of (1.1).

Remark 1.7 The same result also holds if $f$ is nondecreasing, $f(u) / u$ is nondecreasing in a neighbourhood of $+\infty$ and (1.2) holds.

In dimension $D=1$, a necessary and sufficient condition for uniqueness can be derived. Namely, we have the following

Proposition 1.8 Assume that $f$ satisfies the Sharpened Keller-Osserman condition. Then (1.1) admits a unique solution on $\Omega=]-R, R[$ if and only if the equation

$$
\Phi(\alpha)=R
$$

admits exactly one solution.

As a straightforward consequence, we obtain

Corollary 1.9 Assume that $f$ satisfies the Sharpened Keller-Osserman condition. (1.1) admits a unique solution on any domain $\Omega=]-R, R[$ if and only if $\Phi$ : $(0, \infty) \rightarrow(0, \infty)$ is one-to-one.

Remark 1.10 In particular, if $f$ is nondecreasing, one can easily show that $\Phi$ is one-to-one.

\section{Existence of boundary blow-up solutions}

\subsection{Minimality principle}

We restate the well-known sub and supersolution method (see [5] and [6]) and derive elementary but important corollaries.

Proposition 2.1 Consider $\Omega$ a bounded domain of $\mathbb{R}^{D}$ such that all boundary points are regular, $f \in C(\mathbb{R})$ and $g \in C(\partial \Omega)$. Assume there exist two functions $\underline{u}, \bar{u} \in C(\bar{\Omega})$ such that $\underline{u} \leq \bar{u}$ and

$$
\left\{\begin{aligned}
\Delta \underline{u} \geq f(\underline{u}) \text { in } \mathcal{D}^{\prime}(\Omega), & \left(\text { resp. } \Delta \bar{u} \leq f(\bar{u}) \text { in } \mathcal{D}^{\prime}(\Omega)\right) \\
\underline{u} \leq \text { g on } \partial \Omega, & (\text { resp. } \bar{u} \geq \text { g on } \partial \Omega) .
\end{aligned}\right.
$$


Then problem

$$
\left\{\begin{array}{r}
\Delta u=f(u) \text { in } \mathcal{D}^{\prime}(\Omega), \\
u=g \text { on } \partial \Omega .
\end{array}\right.
$$

possesses at least one solution $u \in C(\bar{\Omega}, \mathbb{R})$ such that $\underline{u} \leq u \leq \bar{u}$.

Corollary 2.2 (Minimality Principle) Make the same assumptions as in Proposition 2.1. Then there exists a unique solution $u \in C(\bar{\Omega})$ of $(2.5)$ such that $\underline{u} \leq u$ and $\left.u\right|_{\omega} \leq \bar{v}$ for any open subset $\omega$ of $\Omega$ and any function $\bar{v} \in C(\bar{\omega})$ satisfying

$$
\left\{\begin{array}{r}
\Delta \bar{v} \leq f(\bar{v}) \text { in } \mathcal{D}^{\prime}(\omega), \\
\bar{v} \geq \underline{u} \text { in } \omega, \\
\bar{v} \geq u \text { on } \partial \omega .
\end{array}\right.
$$

We call $u$ the minimal solution of $(2.5)$ relative to $\underline{u}$.

A complete proof of Corollary 2.2 is given in the Appendix. We present here a short proof in the case where $f$ is a locally Lipschitz function.

Proof. Uniqueness : Let $u_{1}, u_{2}$ be two such solutions. Choosing $\omega=\Omega$ and $\bar{v}=u_{2}$ in the statement of Corollary 2.2, we conclude that $u_{1} \leq u_{2}$. Reversing the roles of $u_{1}$ and $u_{2}$, we conclude that $u_{1}=u_{2}$.

Existence: Let $\Lambda=\sup _{[\min \underline{u}, \max \bar{u}]}\left|f^{\prime}\right|, u_{0}=\underline{u}$ and for $k \geq 1$, define $u_{k} \in C(\bar{\Omega})$ inductively by

$$
\left\{\begin{array}{r}
\Delta u_{k}-\Lambda u_{k}=f\left(u_{k-1}\right)-\Lambda u_{k-1} \text { in } \mathcal{D}^{\prime}(\Omega), \\
u_{k}=g \text { on } \partial \Omega .
\end{array}\right.
$$

Then it is known that the sequence $\left(u_{k}\right)$ is nondecreasing and converges to a solution $u \in C^{2}(\Omega) \cap C(\bar{\Omega})$ of $(2.5)$, which satisfies in addition $\underline{u} \leq u \leq \bar{u}$.

Let $\bar{v} \in C(\bar{\omega})$ verify $(2.6)$ and assume by contradiction that the set $\omega_{1}:=\{x \in$ $\omega: \bar{v}(x)<u(x)\}$ is non empty. Clearly $\omega_{1}$ is open. Working, if necessary, with a connected component of $\omega_{1}$, we assume that $\omega_{1}$ is connected. We prove by induction that $\bar{v} \geq u_{k}$ in $\omega_{1}$ for all $k \in \mathbb{N}$. Passing to the limit as $k \rightarrow \infty$, we then obtain a contradiction with the definition of $\omega_{1}$.

By assumption, $\bar{v} \geq \underline{u}=u_{0}$ in $\omega_{1}$. Given $k \geq 1$, assume that $\bar{v} \geq u_{k-1}$ in $\omega_{1}$. In particular, we have that $\bar{v}(x) \in[\min \underline{u}, \max \bar{u}]$ for $x \in \omega_{1}$.

Observe that if $x \in \partial \omega_{1}$ then either $\bar{v}(x)=u(x)$, or $x \in \partial \omega$, whence $\bar{v}(x) \geq u(x)$. Since $u \geq u_{k}$, we conclude that $\bar{v} \geq u \geq u_{k}$ on $\partial \omega_{1}$. Hence,

$$
\left\{\begin{array}{r}
\Delta\left(\bar{v}-u_{k}\right)-\Lambda\left(\bar{v}-u_{k}\right) \leq f(\bar{v})-f\left(u_{k-1}\right)-\Lambda\left(\bar{v}-u_{k-1}\right) \leq 0 \text { in } \mathcal{D}^{\prime}\left(\omega_{1}\right) \\
\bar{v}-u_{k} \geq 0 \text { on } \partial \omega_{1}
\end{array}\right.
$$

By the (weak) Maximum Principle, $\bar{v} \geq u_{k}$ in $\omega_{1}$.

Remark 2.3 Applying the Minimality Principle to (2.5) with nonlinearity $-f$, we also obtain the existence and uniqueness of a maximal solution relative to $\bar{u}$, defined in a straightforward way. 
Remark 2.4 Assume $\Omega=B_{R}$ is a ball centered at the origin and $g$ is a positive constant. If $\underline{u}$ is radial, one easily sees that the minimal solution $u$ relative to $\underline{u}$ is radial : just apply the Minimality Principle 2.2 with $\bar{v}(x)=u(O(x))$, where $O \in \mathcal{O}_{D}$ is an arbitrary rotation of the Euclidean space. A well-known result of Gidas-NiNirenberg [9] states that any solution $u>g$ is radially symmetric, provided $f$ is e.g. locally Lipschitz.

Finally, letting $\phi(r)=u(x)$ for $r=|x|$, it follows from standard ODE theory that $\phi^{\prime}(0)=0$ and $\phi^{\prime}(r)>0$ in $(0, R)$.

Corollary 2.5 (Minimality Principle for blow-up solutions) Let $\Omega$ be a bounded domain of $\mathbb{R}^{D}$ such that all boundary points are regular, $f \in C(\mathbb{R})$. Assume there exist a function $\underline{u} \in C(\bar{\Omega})$ such that $\Delta \underline{u} \geq f(\underline{u})$ in $\mathcal{D}^{\prime}(\Omega)$ and a function $v \in C(\Omega)$ such that $\Delta v \leq f(v)$ in $\mathcal{D}^{\prime}(\Omega), \lim _{x \rightarrow x_{0}} v(x)=+\infty$ for all $x_{0} \in \partial \Omega$ and $v \geq \underline{u}$. Then there exists a unique solution $u \in C(\Omega)$ of (1.1) such that $\underline{u} \leq u$ and $\left.u\right|_{\omega} \leq \bar{v}$ for any open subset $\omega \subset \Omega$ and any $\bar{v} \in C(\omega)$ satisfying

$$
\left\{\begin{aligned}
\Delta \bar{v} & \leq f(\bar{v}) & \text { in } \mathcal{D}^{\prime}(\omega), \\
\bar{v} & \geq \underline{u} & \text { in } \omega, \\
\lim _{x \rightarrow x_{0}} \bar{v}(x) & =+\infty & \text { for all } x_{0} \in \partial \omega .
\end{aligned}\right.
$$

We call $u$ the minimal solution of (1.1) relative to $\underline{u}$.

For a proof, see the Appendix.

Remark 2.6 In contrast to Remark 2.3, there does not exist in general a maximal boundary blow-up solution of (1.1). See Section 6.3 for enlightening counterexamples.

\subsection{Existence of solutions on some ball}

In this section, we prove that (1.2) implies the existence of a boundary blow-up solution on some ball. First, we state and prove a useful technical lemma

Lemma 2.7 Let $\phi \in C^{2}(0, R)$ be a nondecreasing function solving

$$
\phi^{\prime \prime}+\frac{D-1}{r} \phi^{\prime}=f(\phi) \quad \text { in }(0, R) .
$$

Then, given $0<r_{1}<r_{2}<R$,

$$
\frac{1}{\sqrt{2}} \int_{\phi\left(r_{1}\right)}^{\phi\left(r_{2}\right)} \frac{1}{\sqrt{F(s)-F\left(\phi\left(r_{1}\right)\right)}} d s \geq \frac{1}{D-2} r_{1}\left(1-\left(\frac{r_{1}}{r_{2}}\right)^{D-2}\right)
$$

if $D \neq 2$, and

$$
\frac{1}{\sqrt{2}} \int_{\phi\left(r_{1}\right)}^{\phi\left(r_{2}\right)} \frac{1}{\sqrt{F(s)-F\left(\phi\left(r_{1}\right)\right)}} d s \geq r_{1} \ln \frac{r_{2}}{r_{1}}
$$

if $D=2$. 
Proof. For $r \in\left(r_{1}, r_{2}\right),(2.8)$ is equivalent to

$$
\frac{d}{d r}\left(r^{D-1} \phi^{\prime}\right)=r^{D-1} f(\phi) .
$$

Multiplying the above equation by $r^{D-1} \phi^{\prime}$ and integrating between $r_{1}$ and $r$, we obtain

$$
\begin{aligned}
\frac{1}{2} r^{2 D-2}\left(\phi^{\prime}(r)\right)^{2} & \geq \frac{1}{2}\left(r^{2 D-2}\left(\phi^{\prime}(r)\right)^{2}-r_{1}^{2 D-2}\left(\phi^{\prime}\left(r_{1}\right)\right)^{2}\right) \\
& =\int_{r_{1}}^{r} t^{2 D-2} f(\phi(t)) \phi^{\prime}(t) d t \\
& \geq r_{1}^{2 D-2}\left(F(\phi(r))-F\left(\phi\left(r_{1}\right)\right)\right) .
\end{aligned}
$$

So,

$$
\frac{1}{\sqrt{2}} \frac{\phi^{\prime}(r)}{\sqrt{F(\phi(r))-F\left(\phi\left(r_{1}\right)\right)}} \geq\left(\frac{r_{1}}{r}\right)^{D-1} .
$$

Integrating the above equation between $r_{1}$ and $r_{2}$, we obtain the desired result.

Now assume that (1.2) holds for some $\alpha>0$. If $D \neq 2$, assume temporarily that $\Phi(\alpha)<\frac{1}{|D-2|}$. Applying Proposition 2.1 with $\underline{u}=0$ and $\bar{u}=\alpha$, let $u$ be the minimal solution relative to $\underline{u}$ of

$$
\left\{\begin{array}{r}
\Delta u=f(u) \text { in } B_{1}, \\
u=\alpha \text { on } \partial B_{1} .
\end{array}\right.
$$

Using Remark 2.4 and letting $\tilde{\alpha}=u(0), \phi(r):=u(x)$ for $r=|x|$ solves (2.8), subject to the initial conditions $\phi(0)=\tilde{\alpha}$ and $\phi^{\prime}(0)=0$. $\phi$ can thus be extended on some maximal interval $(0, R)$. Assume temporarily that $R<\infty$. Then $u$ is a boundary blow-up solution on $B_{R}$. Indeed, by the definition of $R$, we must have either $\phi(R)=+\infty$ or $\phi^{\prime}(R)=+\infty$. In the latter case, multiply (2.8) by $\phi^{\prime}$ and integrate between 0 and $r$ to obtain that $\frac{1}{2}\left(\phi^{\prime}\right)^{2} \leq F(\phi)$. Hence $F(\phi(R))=+\infty$, $\phi(R)=+\infty$ and $u$ is a boundary blow-up solution. It remains to prove that $R<\infty$.

Assume by contradiction that $R=\infty$. Apply Lemma 2.7 between $r_{1}=1$ and $r_{2}>1$ :

$$
\Phi(\alpha) \geq \frac{1}{D-2}\left(1-\left(\frac{1}{r_{2}}\right)^{D-2}\right)
$$

if $D \geq 3$ and

$$
\Phi(\alpha) \geq \ln r_{2}
$$

if $D=2$. Letting $r_{2}$ converge to $\infty$, we obtain a contradiction if either $D=2$ or $\Phi(\alpha)<\frac{1}{|D-2|}$.

If $D \neq 2$ and $\Phi(\alpha) \geq \frac{1}{|D-2|}$, choose $K>0$ so large that $\frac{1}{K} \Phi(\alpha)<\frac{1}{|D-2|}$. The above proof provides a boundary blow-up solution $u$ of (1.1) on some ball $B_{R}$, when $f$ is replaced by $K^{2} f . \tilde{u}(x):=u(x / K)$ is then a boundary blow-up solution of $(1.1)$ with nonlinearity $f$ on $B_{R K}$. 
Remark 2.8 Let $B$ be a ball of radius $R$ and assume $\underline{u} \in C(\bar{B})$ is such that $\Delta \underline{u} \geq f(\underline{u})$ in $B$. Assume (1.2) holds for some $\alpha \geq \sup _{B} \underline{u}$ and let $\bar{u}=\alpha$. Using Proposition 2.1 , let $u$ be the minimal solution relative to $\underline{u}$ of

$$
\left\{\begin{array}{r}
\Delta u=f(u) \text { in } B, \\
u=\alpha \text { on } \partial B .
\end{array}\right.
$$

Repeating the above proof, we conclude that $u$ can be extended to a radially symmetric boundary blow-up solution on some ball $\tilde{B}$ of radius $\tilde{R}>R$, satisfying $u \geq \underline{u}$ in $B$.

\subsection{Existence of solutions on small balls}

Assume (1.3) holds. By Theorem 1.1, (1.1) has a solution on some ball, and we may define

$$
R_{0}:=\inf \left\{R>0:(1.1) \text { has a solution in } B_{R}\right\}
$$

We assume by contradiction that $R_{0}>0$. Let $\left(\beta_{n}\right)$ be a sequence of real numbers increasing to infinity and satisfying

$$
\lim _{\beta_{n} \rightarrow \infty} \Phi\left(\beta_{n}\right)=0
$$

Applying Proposition 2.1 with $\underline{u}=0$ and $\bar{u}=\beta_{n}$, let $u_{n}$ be the minimal solution relative to $\underline{u}$ of

$$
\left\{\begin{array}{r}
\Delta u_{n}=f\left(u_{n}\right) \text { in } B_{R_{0} / 2}, \\
u_{n}=\beta_{n} \text { on } \partial B_{R_{0} / 2} .
\end{array}\right.
$$

By Remark 2.4, letting $\alpha_{n}=u_{n}(0), \phi_{n}(r):=u_{n}(x)$ for $r=|x|$ solves (2.8) with initial conditions $\phi_{n}(0)=\alpha_{n}$ and $\phi_{n}^{\prime}(0)=0$. By definition of $R_{0}, \phi_{n}$ can be extended so that $\phi_{n}$ remains a solution of $(2.8)$ in $\left(0, R_{0}\right)$. Now apply Lemma 2.7 with $r_{1}=R_{0} / 2$ and $r_{2}=R_{0}$ :

$$
\Phi\left(\beta_{n}\right) \geq \frac{1}{D-2} \frac{R_{0}}{2}\left(1-\left(\frac{1}{2}\right)^{D-2}\right)
$$

if $D \geq 3$, and

$$
\Phi\left(\beta_{n}\right) \geq \frac{R_{0}}{2} \ln (2)
$$

if $D=2$. Passing to the limit as $n \rightarrow \infty$, we obtain a contradiction in both cases. We have just proved that

$$
\inf \left\{R>0:(1.1) \text { has a solution in } B_{R}\right\}=0 \text {. }
$$

Remark 2.9 Let $B$ be a ball of radius $R$ and assume $\underline{u} \in C(\bar{B})$ is such that $\Delta \underline{u} \geq f(\underline{u})$ in $B$. Using Remark 2.8 and working as above, one can show that $\inf \left\{\tilde{R}>\bar{R}:(1.1)\right.$ has a solution $u$ in $B_{\tilde{R}}$ such that $u \geq \underline{u}$ in $\left.B\right\}=R$. 


\subsection{Existence of solutions on smooth domains}

We assume here that (1.3) is valid. Applying Proposition 2.1 with $\underline{u}=0$ and $\bar{u}=N$, $N \in \mathbb{N}$, let $u_{N}$ be the minimal solution relative to $\underline{u}$ of $(2.5)$ with $g \equiv N$. For $x \in \Omega$, choose a ball $B(x, r) \subset \Omega$ such that there exists a boundary blow-up solution $u_{r}$ on $B(x, r)$. This is always possible since (2.10) holds. Applying the Minimality Principle 2.2 with $\bar{v}=u_{r}$, we conclude that $0 \leq u_{N} \leq u_{r}$ in $B(x, r)$. In particular, the sequence $\left(u_{N}\right)$ is uniformly bounded in $B(x, r / 2)$.

Let $K$ denote an arbitrary compact subset of $\Omega$. Covering $K$ by finitely many balls $B\left(x_{i}, r_{i} / 2\right)$, we conclude that $\left(u_{N}\right)$ is uniformly bounded on $K$ by a constant depending only on $K$ and $f$. Applying the Minimality Principle 2.2 with $\bar{v}=u_{N+1}$, we can also infer that $\left(u_{N}\right)$ is a nondecreasing sequence. Using these two facts and elliptic regularity, we conclude that $\left(u_{N}\right)$ converges to a function $u$ solving $\Delta u=f(u)$ in $\Omega$.

Fix a point $x_{0} \in \partial \Omega$ and an arbitrary sequence $\left(x_{k}\right)$ in $\Omega$ converging to $x_{0}$. Then, since $u \geq u_{N}$,

$$
\liminf _{k \rightarrow \infty} u\left(x_{k}\right) \geq \liminf _{k \rightarrow \infty} u_{N}\left(x_{k}\right)=N .
$$

Letting $N$ converge to infinity, we conclude that $u$ is a boundary blow-up solution of $(1.1)$ in $\Omega$.

\subsection{Proof of Theorem 1.1 completed.}

By Section 2.2, we know that if (1.2) holds, there exists a blow-up solution on some ball. Conversely, assume that $u>0$ solves (1.1) on some ball $B$ of radius $R$ centered at the origin. By Corollary 2.5, we may always assume that $u$ is the minimal solution relative to $\underline{u}=0$ of (1.1). In particular $u$ is radial and we define $\phi(r)=u(x)$ for $r=|x|$, so that $\phi$ solves $(2.9)$ in $(0, R)$. Multiplying (2.9) by $r^{D-1} \phi^{\prime}$ and integrating between 0 and $r$, we obtain

$$
\frac{1}{2} r^{2 D-2} \phi^{\prime}(r)^{2}=\int_{0}^{r} t^{2 D-2} f(\phi(t)) \phi^{\prime}(t) d t \leq r^{2 D-2}[F(\phi(r))-F(\phi(0))] .
$$

Integrating once more between 0 and $R$,

$$
0 \leq \int_{0}^{R} \frac{\phi^{\prime}(r)}{\sqrt{2[F(\phi(r))-F(\phi(0))]}} d r \leq R
$$

which implies (1.2) with $\alpha=\phi(0)$.

\subsection{Proof of Theorem 1.4 completed.}

By Section 2.4, we know that if (1.3) holds, there exists a blow-up solution on any domain. Conversely, given $n \in \mathbb{N}$, assume that $u_{n}>0$ solves (1.1) on the ball $B$ of radius $1 / n$ centered at the origin. By Corollary 2.5, we may always assume that $u_{n}$ is the minimal solution relative to $\underline{u}=0$. In particular $u_{n}$ is radial. Let now 
$\beta_{n}=u_{n}(0)$. We claim that $\left(\beta_{n}\right)$ is unbounded. Taking a subsequence if necessary, we then have that $\lim _{n} \beta_{n}=\infty$ and (1.3) follows from (2.11) applied with $R=1 / n$.

It remains to prove that $\left(\beta_{n}\right)$ is unbounded. If not, up to a subsequence, $\left(\beta_{n}\right)$ converges to some $\beta \geq 0$. By (2.11) applied with $R=1 / n$, we have

$$
0 \leq \int_{\beta_{n}}^{\infty} \frac{d t}{\sqrt{2\left[F(t)-F\left(\beta_{n}\right)\right]}} d r \leq 1 / n .
$$

By Fatou's lemma, we conclude that

$$
\int_{\beta}^{\infty} \frac{d t}{\sqrt{2[F(t)-F(\beta)]}} d r=0,
$$

which is not possible.

\section{Asymptotic behaviour of solutions}

\subsection{Blow-up rate of radially symmetric solutions}

Proposition 3.1 Assume that $f$ satisfies the Keller-Osserman condition (1.2). Assume $\phi$ is a radially symmetric and monotone boundary blow-up solution on the unit ball. Then, for $r \sim 1$,

$$
\int_{\phi(r)}^{+\infty} \frac{d t}{\sqrt{F(t)}} \sim \sqrt{2}(1-r)
$$

Proof. Multiplying (2.9) by $r^{D-1} \phi^{\prime}$ and integrating by parts, we easily obtain that given $r \in(0,1)$,

$$
\frac{\left(\phi^{\prime}\right)^{2}(r)}{2}=F(\phi(r))-G_{\phi}(r),
$$

where

$$
G_{\phi}(r)=\frac{2 D-2}{r} \int_{0}^{r}\left(\frac{s}{r}\right)^{2 D-1} F(\phi(s)) d s .
$$

We claim that

$$
G_{\phi}(r)=o(F(\phi(r))), \quad \text { as } r \rightarrow 1 .
$$

Indeed, let $\epsilon>0$. Then, since $F$ is nondecreasing,

$$
\begin{aligned}
& \frac{G_{\phi}(r)}{F(\phi(r))}=\frac{2 D-2}{r} \int_{0}^{1-\epsilon}\left(\frac{s}{r}\right)^{2 D-1} \frac{F(\phi(s))}{F(\phi(r))} d s \\
& +\frac{2 D-2}{r} \int_{1-\epsilon}^{r}\left(\frac{s}{r}\right)^{2 D-1} \frac{F(\phi(s))}{F(\phi(r))} d s . \\
& \leq C \frac{F(\phi(1-\epsilon))}{F(\phi(r))} \quad+C \epsilon \text {. }
\end{aligned}
$$


Letting $r \rightarrow 1$ and then $\epsilon \rightarrow 0$, we obtain the desired result. Returning to (3.13), we obtain

$$
1-\frac{\phi^{\prime}}{\sqrt{2 F(\phi)}}=1-\left[1-\frac{G_{\phi}}{F(\phi)}\right]^{1 / 2}
$$

Combining this with (3.14), it follows that for $r \sim 1$,

$$
1-\frac{\phi^{\prime}}{\sqrt{2 F(\phi)}} \sim \frac{G_{\phi}}{2 F(\phi)}
$$

and, integrating between $r$ and 1 ,

$$
(1-r)-\int_{\phi(r)}^{\infty} \frac{d t}{\sqrt{2 F(t)}} \sim \int_{r}^{1} \frac{G_{\phi}(s)}{2 F(\phi(s))} d s=o(1),
$$

which implies (3.12).

\subsection{Blow-up rate of solutions on smooth domains}

Let $u$ be a blow-up solution on a domain $\Omega$, which satisfies an interior and an exterior sphere condition at any boundary point. Fix $x_{0} \in \partial \Omega$ and let $B_{R} \subset \Omega$ denote a small ball which is tangent to $\partial \Omega$ at $x_{0}$. Fix $\eta \in(0,1)$. Let $\underline{u}:=\left.u\right|_{B_{\eta R}}$. By Remark 2.8, there exists a radial boundary blow-up solution $v$ defined on some ball $\tilde{B} \supset B_{\eta R}$, such that $v \geq \underline{u}$ in $B_{\eta R}$. Let $K>0$ such that $K \tilde{B}=B_{R}$ and let $v_{K}(x):=v\left(\left(x-x_{1}\right) / K+x_{1}\right)$, where $x_{1}$ is the center of $B_{R}$. Then $v_{K}$ solves

$$
\begin{array}{r}
\Delta v_{K}=\frac{1}{K^{2}} f\left(v_{K}\right) \text { in } B_{R}, \\
v_{K}=+\infty \text { on } \partial B_{R} .
\end{array}
$$

Since $u\left(x_{1}\right) \leq v\left(x_{1}\right)=v_{K}\left(x_{1}\right)$, Proposition 3.1 implies that

$$
K \int_{u\left(x_{1}\right)}^{+\infty} \frac{d t}{\sqrt{F(t)}} \geq K \int_{v_{K}\left(x_{1}\right)}^{+\infty} \frac{d t}{\sqrt{F(t)}} \sim \sqrt{2} R .
$$

Letting $R \rightarrow 0$, we then have

$$
K \liminf _{x \rightarrow x_{0}} \frac{\int_{u(x)}^{+\infty} \frac{d t}{\sqrt{F(t)}}}{\delta(x)} \geq \sqrt{2} .
$$

By Remark 2.9, we may take $K$ arbitrarily close to $1 / \eta$. Also, $0<\eta<1$ was chosen arbitrarily. So, letting $K, \eta \rightarrow 1$, we finally obtain

$$
\liminf _{x \rightarrow x_{0}} \frac{\int_{u(x)}^{+\infty} \frac{d t}{\sqrt{F(t)}}}{\delta(x)} \geq \sqrt{2} .
$$

Choose another ball $B_{R^{\prime}} \subset \mathbb{R}^{N} \backslash \bar{\Omega}$ which is tangent to $\partial \Omega$ at $x_{0}$ and a concentric ball $B_{R^{\prime \prime}}$ with $R^{\prime \prime}>R^{\prime}$ so large that $\Omega \subset B_{R^{\prime \prime}}$. Finally, let $A=B_{R^{\prime \prime}} \backslash B_{R^{\prime}}$. Let 
$v$ denote the minimal boundary blow-up solution (relative to $\underline{u}=0$ ) on $A$. By the Minimality Principle 2.5, we deduce that $u \geq v$ in $\Omega$. Applying Proposition 3.1 (which still holds on an annulus) with $v$, we conclude that

$$
\limsup _{x \rightarrow x_{0}} \frac{\int_{u(x)}^{+\infty} \frac{d t}{\sqrt{F(t)}}}{\delta(x)} \leq \limsup _{x \rightarrow x_{0}} \frac{\int_{v(x)}^{+\infty} \frac{d t}{\sqrt{F(t)}}}{\delta(x)} \leq \sqrt{2} .
$$

This finishes the proof of Theorem 1.5. More can be said about the asymptotic behaviour of solutions provided $F$ satisfies some extra growth assumption :

Lemma 3.2 Let $u, v$ denote two radially symmetric boundary blow-up solutions defined on the unit ball $B$. Assume there exist $\beta>0$ and $M>0$ such that $\frac{F(v)}{v^{2}} \geq$ $\beta^{2} \frac{F(u)}{u^{2}}$ whenever $M \leq u \leq v$. Then $u(r) \sim v(r)$ on $\partial B$.

Proof. We recall from the proof of of Proposition 3.1 that for $\phi$ a radially symmetric boundary blow up solution and for $r \sim 1$,

$$
(1-r)-\int_{\phi(r)}^{\infty} \frac{d t}{\sqrt{2 F(t)}} \leq C \int_{r}^{1} \frac{G_{\phi}(s)}{F(\phi(s))} d s \leq C \int_{r}^{1} \frac{\phi(s)}{\sqrt{F(\phi(s))}} d s .
$$

Using $\frac{\phi \dot{\phi}(s)}{\sqrt{2 F(\phi(s))}} \sim 1$, we then obtain that, introducing $K(r)$ such that $1-r=$ $\int_{K(r)}^{\infty} \frac{d t}{\sqrt{2 F(t)}}$,

$$
\int_{K(r)}^{\phi(r)} \frac{d t}{\sqrt{2 F(t)}} \leq C \int_{\phi(r)}^{\infty} \frac{t}{F(t)} d t
$$

Since $F$ is increasing, we thus obtain

$$
\left(1-\frac{K(r)}{\phi(r)}\right) \leq C \frac{\sqrt{F(\phi(r))}}{\phi(r)} \int_{\phi(r)}^{\infty} \frac{t}{F(t)} d t .
$$

Since $\frac{F(v)}{v^{2}} \geq \beta^{2} \frac{F(u)}{u^{2}}$ for $u \leq v$ large enough, (3.16) implies that

$$
\left(1-\frac{K(r)}{\phi(r)}\right) \leq \frac{C}{\beta} \int_{\phi(r)}^{\infty} \frac{d t}{\sqrt{F(t)}}
$$

The classical Keller-Osserman (1.2) condition gives the result.

Corollary 3.3 Assume either that $f$ is convex on some interval $[a,+\infty)$ or that $f(t) / t$ is nondecreasing on $[a,+\infty)$. Then the result of the previous lemma holds.

Proof. Assume $f$ is convex in $[a,+\infty)$ and let $G(t)=F(t+a)-F(a)-f(a) t$. Then $G(0)=G^{\prime}(0)=0$ and $G^{\prime}$ is convex in $\mathbb{R}^{+}$. So $G(t) / t^{2}$ is nondecreasing, i.e.

$$
t \rightarrow \frac{F(t+a)-L(t)}{t^{2}}
$$


is nondecreasing, where $L(t)=F(a)+f(a) t$ is affine. Observe that $\lim _{t \rightarrow \infty} F(t) / t^{2}=$ $+\infty$ since the Keller-Osserman condition (1.2) implies

$$
\frac{u}{2 \sqrt{F(u)}} \leq \int_{u / 2}^{u} \frac{d t}{\sqrt{F(t)}}=o(1) \quad \text { as } u \rightarrow \infty .
$$

It follows that there exists $\beta>0$ such that $\frac{F(v)}{v^{2}} \geq \beta^{2} \frac{F(u)}{u^{2}}$ for $u \leq v$ large enough and we may apply Lemma 3.2. The case where $f(t) / t$ is nondecreasing on $[a,+\infty)$ is similar, so we skip it.

\section{The uniqueness result}

Lemma 4.1 Assume that $f$ is nondecreasing on $[0,+\infty)$ and convex in a neighborhood of $+\infty$ (say $[a,+\infty))$. Consider two radially symmetric boundary blow-up solutions such that $u(r) \leq v(r)$ on $B(0, R)$. Then, in fact, $u=v$ everywhere.

Proof. Set $R=1$ for the sake of simplicity. Since $f$ is nondecreasing, either $u(0)=$ $v(0)$ (and then $u=v$ everywhere) or $u(r)<v(r)$ everywhere since $\partial\left(r^{D-1}(\dot{v}-\dot{u})\right)=$ $r^{D-1}(f(v)-f(u))$, so the map $r \rightarrow v(r)-u(r)$ is nondecreasing. Assume then $u(0)<v(0)$.

Let $\varepsilon>0$. Consider the set $\omega_{\varepsilon}=\{r \in[0,1) ; \forall s<r,(1+\varepsilon) u(s)<v(s)\}$. If $\varepsilon$ is small enough, $0 \in \omega_{\varepsilon}$. Due to lemma 3.2, $R=1 \notin \omega_{\varepsilon}$ since $u \sim v$ close to the boundary. Then introduce $r_{\varepsilon}^{0}=\sup \omega_{\varepsilon}$, which satisfies $0<r_{\varepsilon}^{0}<1$. We now have

$$
v(0)-u(0) \leq v\left(r_{\varepsilon}^{0}\right)-u\left(r_{\varepsilon}^{0}\right)=\varepsilon u\left(r_{\varepsilon}^{0}\right) .
$$

Then either $r_{\varepsilon}^{0}$ converges to $R=1$ when $\varepsilon \rightarrow 0$ or, letting $\varepsilon \rightarrow 0, u(0)=v(0)$ and the proof is over.

Now introduce $a$ such that $f$ is convex on $[a,+\infty)$. Introduce $R_{0}$ such that $u(r) \geq a$ for $r \geq R_{0}$. Then for $\varepsilon$ small enough, $r_{\varepsilon}^{0}>R_{0}$. Set $w(r)=(1+\varepsilon) u(r)$. Then, on the annulus $R_{0}<s<r_{\varepsilon}^{0}$, using the convexity

$$
\begin{aligned}
\Delta(v-w) & =f(v)-(1+\varepsilon) f(u) \\
& \geq f(v)-(1+\varepsilon)\left(f(w)-f(a) \frac{u-a}{w-a}-(1+\varepsilon) f(a)\right. \\
& \geq f(v)-f(w)+\frac{\varepsilon}{w-a}(a f(w)-w f(a)) .
\end{aligned}
$$

Observe now that the map $X \rightarrow \frac{X f(a)-a f(X)}{X-a}$ is majorized by some constant $C$ for $X \geq a$. Then introducing $\chi$ that satisfies $-\Delta \chi=1$ with homogeneous Dirichlet condition at $R=1(\chi(1)=0)$

$$
\Delta(v-w-C \varepsilon \chi) \geq 0
$$

and by the maximum principle, for any $r, R_{0}<r<r_{\varepsilon}^{0}$, 


$$
v(r)-w(r)-C \varepsilon \chi(r) \leq \max \left(-C \varepsilon \chi\left(r_{\varepsilon}^{0}\right), v\left(R_{0}\right)-w\left(R_{0}\right)-C \varepsilon \chi\left(R_{0}\right)\right) .
$$

Then letting $\varepsilon \rightarrow 0$ we obtain that for any fixed $r$ such that $R_{0}<r<1$,

$$
v(r)-u(r) \leq v\left(R_{0}\right)-u\left(R_{0}\right) .
$$

Since the map $r \rightarrow v(r)-u(r)$ is nondecreasing, this implies that $v(r)-u(r)$ is constant on $\left[R_{0}, 1\right)$. By standard ODE theory, this implies that $v$ and $u$ coincide everywhere on $[0,1]$.

Remark 4.2 Since $f$ is nondecreasing, there exists $\underline{U}, \bar{U}$ the minimal and the maximal boundary blow-up solutions of the problem (the latter can be obtained e.g. as the monotone limit of $u(R)$ as $R \rightarrow 1^{-}$, where $u(R)$ denotes the minimal boundary blow-up solution on $B_{R}$ ). Clearly both $\underline{U}$ and $\bar{U}$ are radial and they coincide by the previous lemma. Since any solution $u$ of the problem must stay between $\underline{U}$ and $\bar{U}$, Theorem 1.6 follows. Alternatively, according to a result of Poretta-Veron [15], any boundary blow-up solution is radially symmetric if $f$ is convex in a neighborhood of $+\infty$, whence again Theorem 1.6 follows from the previous lemma.

Remark 4.3 The previous lemma is still valid if we substitute the assumption $\frac{f(u)}{u}$ increasing in a neighborhood of infinity to the convexity assumption. Since the proofs are easier they are left as an exercise to the reader.

\section{Discrete equations}

We are concerned with finite difference approximations of (1.1) when $D=1$ or $D=2$ on a cube or a ball. After introducing some notation, we observe that both the maximum principle and the minimality principle extend to the case of finite difference operators. We conclude this section with some theoretical error estimates, assuming that $f$ is a nondecreasing function.

\subsection{Finite differences}

To begin with, consider the interval $[-1,1]$ or the unit square $[-1,1]^{2}$. Consider a uniform grid $\Omega_{h}$ which mesh size is $h=\frac{1}{L}$ for some integer $L$. The nodes on the grid are respectively $j h$ if $\mathrm{D}=1,-L \leq j \leq L$, or $(i h, j h)$ if $D=2$, with $-L \leq i, j \leq L$.

The discrete Laplace operator is then defined on each point/node of the grid respectively by

$$
\left(\Delta_{h} U\right)_{j}=\frac{1}{h^{2}}\left(-2 U_{j}+U_{j+1}+U_{j-1}\right)
$$

if $D=1$, and

$$
\left(\Delta_{h} U\right)_{i, j}=\frac{1}{h^{2}}\left(-4 U_{i, j}+U_{i, j+1}+U_{i, j-1}+U_{i+1, j}+U_{i-1, j}\right)
$$


if $D=2$. In the above, $U$ is a vector in $\mathbb{R}^{2 L+1}$ (or $\mathbb{R}^{(2 L+1) 2}$ in $2 \mathrm{D}$ ) with components $U_{j} \simeq u(j h)\left(\right.$ or $\left.U_{i, j} \simeq u(i h, j h)\right)$. If $D=2$, we then solve $\Delta_{h} U=f(U)$, i.e. $\left(\Delta_{h} U\right)_{i, j}=f\left(U_{i, j}\right)$ for all interior nodes $(i, j)$ and set $U_{ \pm L, j}=U_{i, \pm L}=N$ at all boundary nodes, where $N$ is a fixed large constant. We work accordingly when $D=1$.

It is standard to prove that the matrix $\Delta_{h}$ has positive inverse, i.e. the entries of the inverse matrix are positive. Therefore, the maximum principle is valid (see [4]). Actually, if $U$ satisfies $\Delta_{h} U \leq 0$ on the interior nodes of the grid and $U \geq 0$ on the boundary, then $U \geq 0$ everywhere. Here and throughout this section we write $U \geq 0$ iff $U_{i, j} \geq 0$ for all $(i, j)$ nodes of the grid. We shall use the same notation for $B$ a matrix: $B \geq 0$ iff the entries of $B$ are all non negative.

When working on the unit ball, we use a slightly modified scheme. Focusing on radially symmetric functions, we approximate the equation

$$
\frac{1}{r} \frac{\partial}{\partial r}\left(r \frac{\partial u}{\partial r}\right)=f \quad \text { for } r \in(0,1)
$$

Discretize $[0,1]$ by setting $L h=1,0 \leq j \leq L$. At $j=L$ set $U_{L}=N$ (boundary condition). For $0<j<L$ solve

$$
\frac{1}{r} \partial(r \partial u) \simeq \frac{1}{j h} D^{+}\left(j h D^{-} U\right)=\frac{-2 U_{j}+U_{j+1}+U_{j-1}}{h^{2}}+\frac{U_{j+1}-U_{j}}{j h^{2}}=F_{j},
$$

where $\left(D^{+} U\right)_{j}=\frac{U_{j+1}-U_{j}}{h},\left(D^{-} U\right)_{j}=\frac{U_{j}-U_{j-1}}{h}$. It remains to define the equation at $j=0$. For that purpose, we use the symmetry property $u(h)=u(-h)$ and the approximation $\frac{\dot{u}(0)}{0}=\ddot{u}(0)$ to set

$$
\frac{4}{h^{2}}\left(-U_{0}+U_{1}\right)=F_{0}
$$

This approximation of the Laplace operator satisfies the maximum principle. Indeed, it can be easily checked that if $F_{j} \geq 0$ then $j \rightarrow U_{j}$ is increasing. The maximum principle follows promptly.

\subsection{Computing an approximation}

We aim to solve the following problem

$$
\begin{array}{r}
\Delta_{h} u_{h}=f\left(u_{h}\right) \text { in } \Omega, \\
u_{h}=N \text { on } \partial \Omega,
\end{array}
$$

for $N$ large enough.

We expect that $u_{h}$ is an approximation for $u$, the minimal boundary blow up solution corresponding to $\underline{u}=0$. As in Proposition 2.1, $u_{h}$ is obtained by monotone iteration, starting from the discrete subsolution 0. We claim that Proposition 2.1 and Corollary 2.2 are valid for the finite difference approximation. The proof follows the guidelines of the continuous case and is left as an exercise to the reader. 
An approximation of the solution to problem (5.26) is recursively obtained by the following discrete iterative scheme:

Consider $u^{k} \in \mathbb{R}^{(2 L+1) D}$, where $L=\frac{1}{h}$, recursively defined by

$$
\begin{aligned}
& u^{0}=0 \quad \text { and for } k \geq 0, \\
& \left\{\begin{array}{l}
\left(\Delta_{h}-\Lambda_{N} I d\right) u^{k+1}=f\left(u^{k}\right)-\Lambda_{N} u^{k} \quad \text { in } \Omega_{h} \\
u^{k+1}=N \quad \text { on } \partial \Omega_{h}
\end{array}\right.
\end{aligned}
$$

where $\Lambda_{N}=\sup _{[0, N]} f^{\prime}$.

Therefore the error between $u$, the minimal boundary blow up solution, and $u^{k}$ the $k^{t h}$ iterate of (5.27) can be split as follows:

$$
I_{h}(u)-u^{k}=I_{h}\left(u-u_{N}\right)+\left(I_{h}\left(u_{N}\right)-u_{h}\right)+\left(u_{h}-u^{k}\right)
$$

where $I_{h}$ is the interpolation operator defined by $I_{h}(u)_{i}=u\left(x_{i}\right)$ when $D=1$ (respectively by $I_{h}(u)_{a}=u(a)$ when $D=2$ for a node $a=(i h, j h)$ on the grid), and $u_{N}$ is the solution of

$$
\left\{\begin{aligned}
\Delta u & =f(u) \text { in } \Omega \\
u & =N \text { on } \partial \Omega
\end{aligned}\right.
$$

\subsection{Error estimate}

Throughout this section, we assume that $f$ is a convex increasing function and that $\Omega=[-1,1]^{D}$. We first bound from above the rate of convergence of the algorithm $(5.27)$.

Lemma 5.1 Let $u^{k} \in \mathbb{R}^{(2 L+1) D}$ be given by (5.27) and $u_{h}=\lim _{k \rightarrow \infty} u^{k}$. Then, there exist constants $C=C(D), \mu=\mu(D)>0$ and $\lambda=\lambda(f)<\Lambda_{N}$ such that

$$
\left\|u^{k}-u_{h}\right\|_{\ell^{\infty}} \leq C\left(1-\frac{\lambda}{\Lambda_{N}}\right)^{k} \min \left(1, \frac{1}{h^{D / 2}}\left[1-\frac{\mu}{\Lambda_{N}}+\frac{\mu^{2}}{\Lambda_{N}^{2}}\right]^{k}\right)\left\|u^{0}-u_{h}\right\|_{\ell^{\infty}} .
$$

Proof. In the sequel let us denote by $a$ a node of the grid (that is $a=i h$ in $1 \mathrm{D}$ or $a=(i h, j h)$ in $2 \mathrm{D}) .\left(u^{k}\right)$ is a nondecreasing sequence in $\mathbb{R}^{(2 L+1) D}$ (i.e. $u_{a}^{k} \leq u_{a}^{k+1}$ for each node $a)$. By the mean value theorem, there exists $\theta_{a} \in\left(u_{a}^{k},\left(u_{h}\right)_{a}\right)$ such that

$$
f\left(u_{a}^{k}\right)-f\left(\left(u_{h}\right)_{a}\right)=f^{\prime}\left(\theta_{a}\right)\left(u_{a}^{k}-\left(u_{h}\right)_{a}\right)
$$

Therefore

$$
\left|\left(\left(u_{h}\right)_{a}-\frac{f\left(\left(u_{h}\right)_{a}\right)}{\Lambda_{N}}\right)-\left(\left(u^{k}\right)_{a}-\frac{f\left(\left(u^{k}\right)_{a}\right)}{\Lambda_{N}}\right)\right| \leq\left(1-\frac{\lambda}{\Lambda_{N}}\right)\left(\left(u_{h}\right)_{a}-u_{a}^{k}\right),
$$

where $\lambda=\inf f^{\prime}$. 
On the other hand,

$$
u_{h}-u^{k}=\left(I d-\frac{\Delta_{h}}{\Lambda_{N}}\right)^{-1}\left(\left(u_{h}-\frac{f\left(u_{h}\right)}{\Lambda_{N}}\right)-\left(u^{k}-\frac{f\left(u^{k}\right)}{\Lambda_{N}}\right)\right),
$$

where $f(u)$ denotes the vector with components $f(u)_{a}=f\left(u_{a}\right)$. The key argument is to observe that the matrix $I d-\frac{\Delta_{h}}{\Lambda_{N}}$ satisfies the maximum principle. Therefore,

$$
0 \leq u_{h}-u^{k+1} \leq\left(1-\frac{\lambda}{\Lambda_{N}}\right)\left(I d-\frac{\Delta_{h}}{\Lambda_{N}}\right)^{-1}\left(u_{h}-u^{k}\right),
$$

where inequalities hold component by component. We thus obtain that

$$
\left\|u_{h}-u^{k}\right\|_{\ell^{\infty}} \leq\left(1-\frac{\lambda}{\Lambda_{N}}\right)^{k}\left\|\left(I d-\frac{\Delta_{h}}{\Lambda_{N}}\right)^{-k}\right\|_{\mathcal{L}\left(\ell^{\infty}\right)}\left\|u_{h}-u^{0}\right\|_{\ell^{\infty}} .
$$

On the one hand, the maximum principle implies that

$$
\left\|\left(I d-\frac{\Delta_{h}}{\Lambda_{N}}\right)^{-k}\right\|_{\mathcal{L}(\ell \infty)} \leq C
$$

for some constant $C$ depending only on the dimension $D$.

Also, since the spectrum of $-\Delta_{h}$ lies in a segment $\left[\mu, \frac{\mu}{h^{2}}\right]$ (see [4]) and $\frac{1}{\sqrt{(2 / h+1) D}} \|$. $\left\|_{\ell^{2}} \leq\right\| \cdot\left\|_{\ell^{\infty}} \leq\right\| \cdot \|_{\ell^{2}}$ in $\mathbb{R}^{(2 L+1) D}$, we get

$$
\left\|\left(I d-\frac{\Delta_{h}}{\Lambda_{N}}\right)^{-k}\right\|_{\mathcal{L}(\ell \infty)} \leq C h^{-D / 2}\left\|\left(I d-\frac{\Delta_{h}}{\Lambda_{N}}\right)^{-k}\right\|_{\mathcal{L}\left(\ell^{2}\right)}=C h^{-D / 2}\left(\frac{1}{1+\frac{\mu}{\Lambda_{N}}}\right)^{k}
$$

Using that $\frac{1}{1+\frac{\mu}{\Lambda_{N}}} \leq 1-\frac{\mu}{\Lambda_{N}}+\frac{\mu^{2}}{\Lambda_{N}^{2}}$ and collecting (5.35), (5.36) and (5.37), the proof is over.

We now provide an upper bound for $u_{h}-I_{h}\left(u_{N}\right)$.

Lemma 5.2 Assume $f$ is convex. Let $u_{h}$ be the solution of (5.26) and $u_{N}$ be the solution of (5.29). Then,

$$
\left\|u_{h}-I_{h}\left(u_{N}\right)\right\|_{\ell^{\infty}} \leq C h^{2} \alpha(N, f)
$$

where $\alpha(N, f)=\left\|u_{N}^{(4)}\right\|_{L^{\infty}}$ is a constant depending only on $N$ and $f$.

Proof. For the sake of simplicity we will denote $I_{h}\left(u_{N}\right)$ by $u_{N}$, this introduces no confusion. 
We write down the proof for the $2 \mathrm{D}$ problem, leaving the $1 \mathrm{D}$ case as an exercise to the reader. Let $a=(i h, j h)$ be a node on the grid. By the mean value theorem, for each node $a$, there exist $\xi, \eta$ in $\mathbb{R}^{2}$ such that

$$
|\xi-a|<h \text { and }|\eta-a|<h,
$$

and

$$
\Delta_{h}\left(u_{N}\right)_{a}-\left(\Delta u_{N}\right)_{a}=\operatorname{ch}^{2}\left(\frac{\partial^{4} u}{\partial x^{4}}(\xi)+\frac{\partial^{4} u}{\partial y^{4}}(\eta)\right)
$$

Therefore

$$
\left\{\begin{array}{l}
\Delta_{h}\left(u_{h}-u_{N}\right)_{a}=f\left(u_{h}\right)_{a}-f\left(u_{N}\right)_{a}-c h^{2}\left(\frac{\partial^{4} u}{\partial x^{4}}(\xi)+\frac{\partial^{4} u}{\partial y^{4}}(\eta)\right) \text { in } \Omega_{h} \\
\left(u_{h}-u_{N}\right)_{a}=0 \text { on } \partial \Omega_{h} .
\end{array}\right.
$$

Consider $\omega=u_{h}-u_{N}$. Then working as in the previous lemma, we obtain

$$
w_{a}=\left[\left(\Delta_{h}+\Lambda_{N}\right)^{-1}\left(I d-D\left(\frac{f^{\prime}(\theta)}{\Lambda_{N}}\right)\right) \Lambda_{N}\right] \operatorname{ch}^{2}\left(\frac{\partial^{4} u}{\partial x^{4}}(\xi)+\frac{\partial^{4} u}{\partial y^{4}}(\eta)\right)
$$

where $\theta_{a} \in\left(u_{N}(a),\left(u_{h}\right)_{a}\right)$.

We therefore obtain

$$
\left\|w_{a}\right\|_{\ell^{\infty}} \leq \frac{1}{\Lambda_{N}} \frac{4}{1+\frac{4}{\Lambda_{N}}}\left\|\Delta_{h}^{-1}\right\|_{\mathcal{L}(\ell \infty)} C h^{2}\left\|u_{N}^{(4)}\right\|_{L^{\infty}}
$$

where $\left\|u_{N}^{(4)}\right\|_{L^{\infty}}=\max _{|\alpha| \leq 4}\left(\left\|\partial^{\alpha} u_{N}\right\|_{L^{\infty}}\right)$.

Remark 5.3 When $D=1$ and $f(u)=u^{p}$ with $p \geq 2$, the constant $\alpha(N, f)$ is given by

$$
\alpha(N, f)=\left\|u_{N}^{(4)}\right\|_{L^{\infty}}=\frac{p(3 p-1)}{p+1} N^{2 p-1}
$$

\subsection{Error estimate for $\left\|u-u_{N}\right\|$}

Assume here that the Sharpened Keller-Osserman condition (1.3) is valid. Consider then a sequence $\left(\alpha_{N}\right)$ such that $\Phi\left(\alpha_{N}\right)$ converges towards 0. Consider the minimal solution $u_{N}$ of

$$
\begin{aligned}
\Delta u_{N} & =f\left(u_{N}\right) \quad \text { in } \Omega=B(0,1), \\
u_{N} & =\alpha_{N} \quad \text { on } \partial \Omega .
\end{aligned}
$$

Then one may wonder how $u_{N}$ approximates the minimal boundary blow up solution $u$ defined on the unit ball. We first state a qualitative result 
Proposition 5.4 There exists $R_{N}$ such that $u_{N}$ is a boundary blow up solution on $B\left(0, R_{N}\right)$. Moreover

$$
R_{N}-1 \sim \frac{1}{\sqrt{2}} \int_{\alpha_{N}}^{+\infty} \frac{d t}{\sqrt{F(t)}}
$$

Remark 5.5 This proposition shows that when we plot the approximation $u_{N}$, we plot in fact a boundary blow-up solution on a ball that is close to the unit ball.

Let us proceed to the proof in the case where $D \geq 3$. The cases $D=1,2$ are very similar and then omitted. Assume that $u_{N}$ extends to $\mathbb{R}^{D}$. Then by Lemma 2.7

$$
\frac{1}{\sqrt{2}} \int_{u_{N}(1)}^{u_{N}(r)} \frac{d t}{\sqrt{F(t)-F\left(u_{N}(1)\right)}} \geq \frac{1}{D-2}\left(1-\left(\frac{1}{r}\right)^{D-2}\right) .
$$

Here we have used that $u_{N}$ is radially symmetric. Therefore since $u_{N}(1)=\alpha_{N}$, $\Phi\left(\alpha_{N}\right) \geq \frac{1}{D-2}\left(1-\left(\frac{1}{r}\right)^{D-2}\right) . \quad N \rightarrow+\infty$ leads to a contradiction. The estimate (5.45) comes from Proposition 3.1.

Remark 5.6 Observe that if $f(u)=u^{p}$, then $u_{N}(r)=R_{N}^{-\frac{2}{p-1}} u\left(r R_{N}\right)$. In that case, $0 \leq u(r)-u_{N}(r) \leq C\left(R_{N}-1\right)(\dot{u}(r)+u(r))$. The inequality is sharp for some numerical constant $C$. To prove the estimate in a more general context, we need extra hypotheses.

Definition 5.7 Consider $g:[0,+\infty) \rightarrow[0,+\infty)$ a function. We say that $g$ is strongly increasing if the function

$$
\rho(\lambda)=\inf _{u \geq 0} \frac{g(\lambda u)}{g(u)}
$$

is a $C^{1}$ increasing function on $[1,+\infty)$ that satisfies $\dot{\rho}(1) \neq 0$.

A strongly increasing function is increasing in the usual sense. $g(u)=u^{p}, p>0$, is strongly increasing; $g(u)=\ln (u+1)$ is not.

We now state and prove

Proposition 5.8 Assume that $\frac{f(u)}{u}$ is strongly increasing. Then

$$
0 \leq u(r)-u_{N}(r) \leq C\left(R_{N}-1\right)(\dot{u}(r)+u(r))
$$

Proof. $v(r)=\lambda u_{N}\left(r R_{N}\right)$ is a blow up function on the unit ball. We have

$$
\Delta v=\lambda R_{N}^{2} f\left(\frac{v}{\lambda}\right) \leq \frac{R_{N}^{2}}{\rho(\lambda)} f(v) .
$$

For $N$ large enough, we choose $\lambda_{N}$ close to 1 such that $\rho\left(\lambda_{N}\right)=R_{N}^{2}$. Then $v$ is a blow-up supersolution to (1.1). Since $u$ is the minimal blow up solution, then $u(r) \leq v(r)$. Therefore 


$$
\begin{array}{r}
0 \leq u(r)-u_{N}(r) \leq u(r)-\frac{1}{\lambda_{N}} v\left(\frac{r}{R_{N}}\right) \leq \\
\left(R_{N}-1\right) \dot{u}(r)+\left(1-\frac{1}{\lambda_{N}}\right) u\left(r R_{N}^{-1}\right) \leq C\left(R_{N}-1\right)(\dot{u}(r)+u(r)),
\end{array}
$$

since $\left(1-\frac{1}{\lambda_{N}}\right) \leq 2 \frac{R_{N}-1}{\dot{\rho}(1)}$.

\section{Numerical computations}

In this section, we present some numerical results obtained with our method of approximation.

Remark 6.1 At this stage, we would like to point out that our method is selfcontained, and does not use the knowledge of the boundary blow up behavior of the solution. In fact, as in [11], one can introduce another approximate problem such as taking $\Omega_{\varepsilon} \subset \Omega$ where $\operatorname{dist}\left(\Omega, \Omega_{\varepsilon}\right) \leq \varepsilon$, and solve the problem

$$
\left\{\begin{array}{r}
\Delta u_{\varepsilon}=f\left(u_{\varepsilon}\right) \text { in } \Omega_{\varepsilon} \\
u_{\varepsilon}(x)=K(x) \text { on } \partial \Omega_{\varepsilon}
\end{array}\right.
$$

where

$$
\frac{1}{\sqrt{2}} \int_{K(x)}^{+\infty} \frac{d t}{\sqrt{F(t)}}=\operatorname{dist}(x, \partial \Omega) .
$$

We discuss our numerical results successively on three examples:

- $f(u)=u^{2}$ (i.e. $\frac{f(u)}{u}$ is increasing),

- $f(u)=u^{2}(2+\cos u)$,

- $f(u)=u^{2}(1+\cos u)$.

6.1 $f(u)=u^{2}$

Since $\frac{f(u)}{u}$ is increasing, on any domain we have a unique boundary blow up solution (see e.g. [1] and references therein).

We see in Figure 1 that $\Phi$ is a strictly decreasing function. 


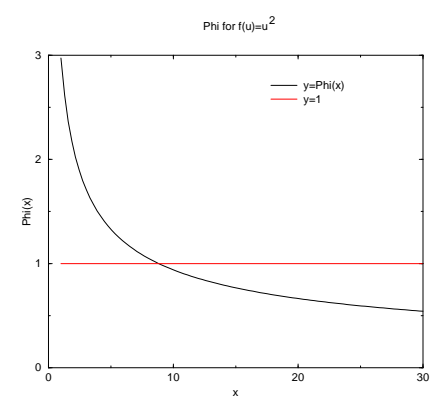

Figure 1: $\Phi$ when $f(u)=u^{2}$

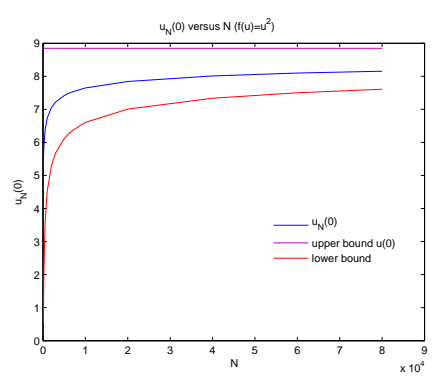

Figure 2: Convergence of $u_{h}(0)$ to $u(0)$ versus $h$

For the sake of completeness, we plot in $1 \mathrm{D}$ the values of $u_{h}(0)$ for several values of $h$ and we compare to theoretical results (see Figure 2 and Proposition 5.8).

Remark 6.2 The value of $u(0)$ is obtained by solving

$$
\frac{1}{\sqrt{2}} \int_{u(0)}^{+\infty} \frac{d t}{\sqrt{F(t)-F(u(0))}}=1
$$

6.2 $f(u)=u^{2}(2+\cos u)$

This function $f$ satisfies the Sharpened Keller-Osserman condition (1.3) (see the Appendix for a proof in a similar case).

Figure 3 below shows that $\Phi(\alpha)$ tends to 0 when $\alpha$ tends to $+\infty$ (then the Sharpened Keller-Osserman condition is valid).

But $\Phi$ is not a decreasing function ; for instance for $c \approx 0.49$ there exist $\alpha \neq \beta$ such that $\Phi(\alpha)=\Phi(\beta)=c$. Therefore, at least in 1D, uniqueness does not hold.

Remark : The uniqueness result for $f(u)=u^{2}(2+\cos u)$ in $B(0,1) \subset \mathbb{R}^{D}$ is still an open question for $D \geq 2$.

We now plot an approximation of the minimal boundary blow-up radial solution on $B(0,1) \subset \mathbb{R}^{2}$. 

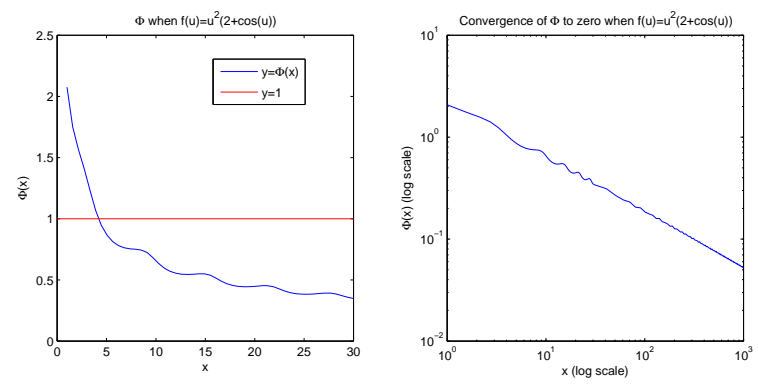

Figure 3: $\Phi$ when $f(u)=u^{2}(2+\cos (u))$

6.3 $f(u)=u^{2}(1+\cos u)$

We first state

Proposition 6.3 The function $f(u)=u^{2}(1+\cos u)$ satisfies the Sharpened KellerOsserman condition (1.3). Moreover,

$$
\lim _{\alpha \rightarrow(2 k+1) \pi} \Phi(\alpha)=+\infty
$$

For a proof, see the Appendix (Proposition 7.1).

Figure 4 shows that for any domain, there exist an infinite sequence of boundary blow up solutions.

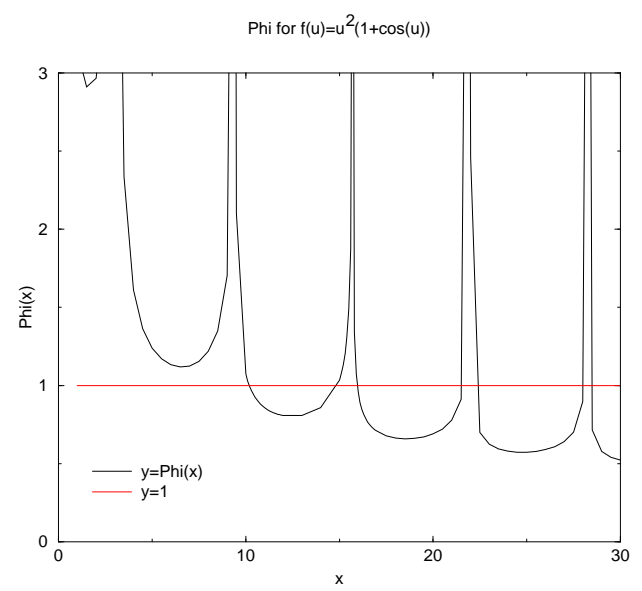

Figure 4: $\Phi$ when $f(u)=u^{2}(1+\cos (u))$

When $D=1$, this follows from the fact that $\Phi(\alpha)=R$ admits an infinite number of solutions $\alpha$. When $D \geq 2$, fix an integer $m$ and observe that $\alpha_{m}=(2 m+1) \pi$ 
is a subsolution. Let $u_{m}$ denote the minimal boundary blow-up solution relative to $\underline{u}=\alpha_{m}$. Infinitely many $u_{m}$ 's must be distinct. Indeed, choose $m_{1}$ such that $\alpha_{m_{1}}>u_{0}(0)$. Then $u_{m_{1}}(0) \geq \alpha_{m_{1}}>u_{0}(0)$. Repeating this process inductively yields infinitely many distinct solutions $u_{m_{k}}$.

We plot in figure 5 approximations of different boundary blow-up solutions on the interval $[-1,1]$.

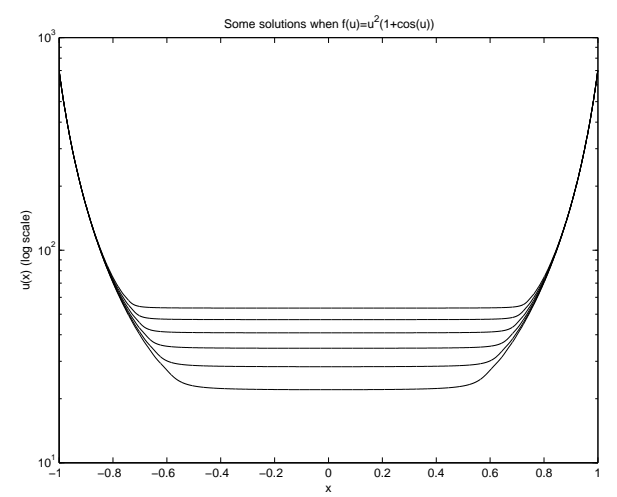

Figure 5: Some solutions when $f(u)=u^{2}(1+\cos u)$

Figure 6 shows two radial approximations of different boundary blow up solutions on the unit ball in $\mathbb{R}^{2}$.

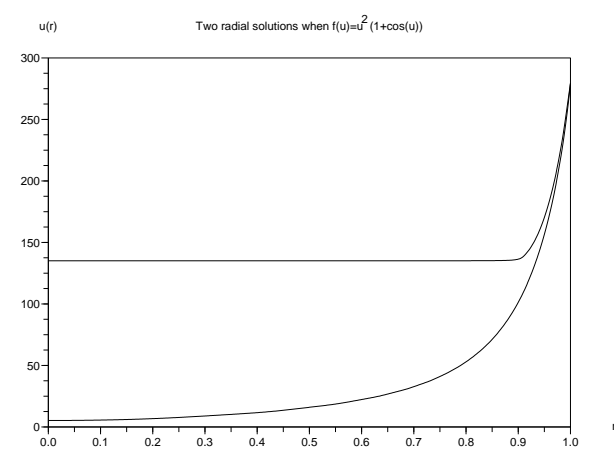

Figure 6: Solutions on the disk when $f(u)=u^{2}(1+\cos u)$ 


\section{Appendix}

\subsection{Regarding the two forms of the Keller-Osserman condi- tion}

We start out by showing that the two forms of the Keller-Osserman condition are equivalent.

Proof of Proposition 1.2. Assume that $f$ satisfies the Keller-Osserman condition. Up to translation, we may always assume that

$$
\int_{0}^{+\infty} \frac{d t}{\sqrt{F(t)}}<+\infty
$$

Consider the change of variable $u=F(t)$. Then, letting $g(u)=\left(F^{-1}\right)^{\prime}(u)$, (7.50) reads also

$$
\int_{0}^{+\infty} \frac{g(u)}{\sqrt{u}} d u<+\infty
$$

whereas (1.3) can be rewritten as

$$
\liminf _{\beta \rightarrow+\infty} \int_{\beta}^{+\infty} \frac{g(u)}{\sqrt{u-\beta}} d u=0 .
$$

First step: we claim that

$$
\limsup _{\beta \rightarrow+\infty} \int_{2 \beta}^{+\infty} \frac{g(u)}{\sqrt{u-\beta}} d u=0 .
$$

Observe that $u \leq 2(u-\beta)$ and then

$$
\int_{2 \beta}^{+\infty} \frac{g(u)}{\sqrt{u-\beta}} d u \leq \sqrt{2} \int_{2 \beta}^{+\infty} \frac{g(u)}{\sqrt{u}} d u .
$$

Second step: it remains to prove that

$$
\liminf _{\beta \rightarrow+\infty} \int_{\beta}^{2 \beta} \frac{g(u)}{\sqrt{u-\beta}} d u=0 .
$$

We argue by contradiction. Let us observe that

$$
\int_{\beta}^{2 \beta} \frac{g(u)}{\sqrt{u-\beta}} d u=\frac{1}{2} \int_{0}^{\sqrt{\beta}} g\left(u^{2}+\beta\right) d u .
$$

Let us assume that there exists $C>0$ such that for any $\beta$

$$
0<C \leq \int_{0}^{\beta} g\left(u^{2}+\beta^{2}\right) d u
$$


Integrate this for $\beta$ between 0 and $R$

$$
0<C R \leq \int_{0}^{R} \int_{0}^{R} g\left(u^{2}+\beta^{2}\right) \chi_{u \leq \beta} d u d \beta .
$$

The right hand side of this inequality is bounded by an integral on an half disc of radius $R$. By symmetry and using polar coordinates

$$
0<C R \leq \pi \int_{0}^{R} g\left(r^{2}\right) r d r=\frac{\pi}{2} \int_{0}^{R^{2}} g(s) d s .
$$

Remember that $g$ is the derivative of $F^{-1}$. Thus,

$$
0<C R \leq \frac{\pi}{2} F^{-1}\left(R^{2}\right) .
$$

Setting $\xi=F^{-1}\left(R^{2}\right)$ this leads to $F(\xi) \leq C \xi^{2}$. This contradicts the KellerOsserman condition (1.2).

Next, we consider the special case $f(u)=u^{2}(1+\cos u)$

Proposition 7.1 Let $f(u)=u^{2}(1+\cos u)$. Then,

$$
\begin{aligned}
& \limsup _{\alpha \rightarrow \infty} \Phi(\alpha)=\infty, \\
& \liminf _{\alpha \rightarrow \infty} \Phi(\alpha)=0 .
\end{aligned}
$$

Proof. Set $\alpha=(2 k+1) \pi$. For $t$ close to $\alpha, F(t)-F(\alpha) \sim \alpha^{2}(t-\alpha)^{2}$. Therefore $\Phi(\alpha)=+\infty$. In particular, $\lim \sup _{\alpha \rightarrow \infty} \Phi(\alpha)=\infty$.

\subsection{Minimality Principle}

We present here the proof of Corollary 2.2 and Corollary 2.5.

Proof of Corollary 2.2. Let $\underline{u}, \bar{u}$ be the sub and supersolution given in the statement of Proposition 2.1. Let $(I,>)$ denote the set of all finite families containing $\bar{u}$ of supersolutions of (2.5) which stay above $\underline{u}$, ordered by inclusion : $i \in I$ if there exist $n \in \mathbb{N}$ and supersolutions $\bar{v}_{k} \in C(\bar{\Omega}), 1 \leq k \leq n$ (i.e. (2.4) holds when $\bar{u}$ is replaced by $\left.\bar{v}_{k}\right)$ with $\bar{v}_{k} \geq \underline{u}$, such that $i=\left\{\bar{u}, \bar{v}_{1}, \ldots, \bar{v}_{n}\right\}$.

$I$ is non-empty since $\{\bar{u}\} \in I . I$ is filtrating increasing, i.e. if $i_{1}, i_{2} \in I$ there exists $i_{3} \in I$ such that $i_{3}>i_{1}, i_{2}$ (take e.g. $i_{3}=i_{1} \cup i_{2}$ ). We prove that given $i=\left\{\bar{u}, \bar{v}_{1}, \ldots, \bar{v}_{n}\right\} \in I$ there exists a solution $u_{i} \in C(\bar{\Omega})$ of (2.5) such that $u_{i} \leq \bar{v}$ for all $\bar{v} \in i$. Let indeed $u^{0}$ denote the solution given by Proposition 2.1. Following [6], since $\underline{u} \leq u^{0} \leq \bar{u}, u^{0}$ is also a solution of (2.5), when $f$ is replaced by the truncation $f^{0} \in C(\bar{\Omega} \times \mathbb{R})$ defined by

$$
f^{0}(x, u)=\left\{\begin{aligned}
f(\underline{u}(x)) & \text { if } u<\underline{u}(x) \\
f(u) & \text { if } \underline{u}(x) \leq u \leq \bar{u}(x) \\
f(\bar{u}(x)) & \text { if } u>\bar{u}(x)
\end{aligned}\right.
$$


In [6], the authors prove that in fact any solution $u$ of (2.5) with nonlinearity $f^{0}$ satisfies $\underline{u} \leq u \leq \bar{u}$ (and solves the problem with the original nonlinearity $f)$. For convenience, we reproduce here the argument of Clément and Sweers : take any solution $u$ of $(2.5)$ with nonlinearity $f^{0}$. Assume by contradiction that $\Omega_{+}:=\{x \in \Omega: u(x)>\bar{u}(x)\}$ is non-empty. Working, if necessary, on a connected component of $\Omega_{+}$, we may also assume that $\Omega_{+}$is connected. For $x \in \partial \Omega_{+}$, either $u(x)=\bar{u}(x)$ or $x \in \partial \Omega$, so that $u(x)=g(x) \leq \bar{u}(x)$. Hence,

$$
\left\{\begin{array}{r}
\Delta(\bar{u}-u) \leq f(\bar{u})-f^{0}(x, u)=0 \text { in } \mathcal{D}^{\prime}\left(\Omega_{+}\right) \\
\bar{u}-u \geq 0 \text { on } \partial \Omega_{+} .
\end{array}\right.
$$

By the (weak) Maximum Principle, $\bar{u} \geq u$ in $\Omega_{+}$, which is a contradiction. Hence, $u \leq \bar{u}$ and we can prove similarly that $u \geq \underline{u}$.

Define now the truncation $f^{1} \in C(\bar{\Omega} \times \overline{\mathbb{R}})$ of $f^{0}$ associated to $\bar{v}_{1}$ by :

$$
f^{1}(x, u)=\left\{\begin{aligned}
f^{0}(x, \underline{u}(x)) & \text { if } u<\underline{u}(x) \\
f^{0}(x, u) & \text { if } \underline{u}(x) \leq u \leq \bar{v}_{1}(x) \\
f^{0}\left(x, \bar{v}_{1}(x)\right) & \text { if } u>\bar{v}_{1}(x)
\end{aligned}\right.
$$

Clearly, $\underline{u}$ and $\bar{v}_{1}$ are a sub and a super solution of (2.5) with nonlinearity $f^{1}$. Applying Proposition 2.1 (which still holds for non-autonomous nonlinearities, see $[6]$ ), we can thus construct a solution $u^{1}$ of $(2.5)$ with nonlinearity $f^{1}$, satisfying $\underline{u} \leq u^{1} \leq \bar{v}_{1}$. Clearly, $u^{1}$ is a solution of the problem with nonlinearity $f^{0}$ and, as we mentioned earlier, we must have $u^{1} \leq \bar{u}$. Repeating the process inductively, we obtain a solution $u_{i}:=u^{n}$ such that $\underline{u} \leq u_{i} \leq \bar{u}, \bar{v}_{1}, \ldots, \bar{v}_{n}$.

Note that $u_{i}$ may not be unique. Nevertheless, using the Axiom of Choice on the set of all such solutions, we can construct a well-defined generalized sequence $\left(u_{i}\right)_{i \in I}$, contained in the set $K$ of all solutions $u$ satisfying $\underline{u} \leq u \leq \bar{u}$.

By standard elliptic estimates, $K$ is a compact subset of $C(\bar{\Omega})$; so there exists a generalized subsequence $\left(u_{\phi(j)}\right)_{j \in J}$ converging to a solution $u$ of $(2.5)$.

Choose now an arbitrary supersolution $\bar{v} \geq \underline{u}$ and let $i_{1}:=\{\bar{v}, \bar{u}\} \in I$. Given $\epsilon>0$, let $j_{0} \in J$ such that $j>j_{0} \Longrightarrow\left\|u_{\phi(j)}-u\right\|_{\infty}<\epsilon$. Also choose $j_{1} \in J$ such that $j>j_{1} \Longrightarrow \phi(j)>i_{1}$. Finally pick $j_{3}>j_{1}, j_{2}$. Then, for $j>j_{3}$,

$$
u \leq\left\|u_{\phi(j)}-u\right\|_{\infty}+u_{\phi(j)} \leq \epsilon+\bar{v}
$$

Letting $\epsilon \rightarrow 0$, we conclude that $u \leq \bar{v}$ for any supersolution $\bar{v} \geq \underline{u}$. Clearly, $u$ is the unique such solution.

It remains to prove that given any subdomain $\omega$ and any function $\bar{v} \in C(\bar{\omega})$ satisfying (2.6), $u \leq \bar{v}$. Fix such a function $\bar{v}$ and define $h^{k} \in C(\bar{\Omega} \times \mathbb{R}), k=0,1$, by

$$
h^{0}(x, t)=\left\{\begin{aligned}
f(\underline{u}(x)) & \text { if } t<\underline{u}(x), \\
f(t) & \text { if } \underline{u}(x) \leq t \leq u(x), \\
f(u(x)) & \text { if } t>u(x)
\end{aligned}\right.
$$


and

$$
h^{1}(x, t)=\left\{\begin{aligned}
h^{1}(x, \underline{u}(x)) & \text { if } t<\underline{u}(x), \\
h^{1}(x, t) & \text { if } x \in \Omega \backslash \omega \text { or if } x \in \omega \text { and } \underline{u}(x) \leq t \leq \bar{v}(x), \\
h^{1}(x, \bar{v}(x)) & \text { if } x \in \omega \text { and } t>\bar{v}(x) .
\end{aligned}\right.
$$

Working as before, we may solve (2.5) with nonlinearity $h^{1}$ and obtain a solution $\tilde{u}$ of $(2.5)$ with nonlinearity $f$ such that $\underline{u} \leq \tilde{u} \leq u$ and $\left.\tilde{u}\right|_{\omega} \leq \bar{v}$. Since $\tilde{u}$ is a (super)solution of (2.5), we also have $u \leq \tilde{u}$. Hence $\left.u\right|_{\omega}=\left.\tilde{u}\right|_{\omega} \leq \bar{v}$.

Proof of Corollary 2.5. Clearly, there exists at most one such solution. Now, let $N$ denote any integer larger than $\|\underline{u}\|_{L^{\infty}(\Omega)}$ and let $u_{N}$ denote the minimal solution relative to $\underline{u}$ of

$$
\left\{\begin{array}{r}
\Delta u_{N}=f\left(u_{N}\right) \text { in } \mathcal{D}^{\prime}(\Omega) \\
u_{N}=N \text { on } \partial \Omega .
\end{array}\right.
$$

Using the Minimality Principle 2.2, one can easily show that the sequence $\left(u_{N}\right)$ is nondecreasing and that $u_{N} \leq N$. Take any (smooth) open set $\omega \subset \subset \Omega$ such that $v \geq N$ on $\partial \omega$. By the Minimality Principle 2.2 again, we conclude that $\left.v\right|_{\omega} \geq\left. u_{N}\right|_{\omega}$. Since this holds for any such $\omega$, we conclude that $v \geq u_{N}$ in $\Omega$. In particular the sequence $\left(u_{N}\right)$ is bounded on compact subsets of $\Omega$ and using elliptic regularity, we conclude that $\left(u_{N}\right)$ converges to a blow-up solution $u$ of (1.1) such that $\underline{u} \leq u \leq v$.

Now take $\omega \subset \Omega$ open and $\bar{v} \in C(\omega)$ satisfying (2.7). Take $\tilde{\omega} \subset \subset \omega$ such that $\bar{v} \geq N$ on $\partial \tilde{\omega}$. Applying the Minimality Principle 2.2, we have that $\left.u_{N}\right|_{\tilde{\omega}} \leq\left.\bar{v}\right|_{\tilde{\omega}}$. Again, since $\tilde{\omega} \subset \subset \omega$ is arbitrary, we conclude that $\left.u_{N}\right|_{\omega} \leq \bar{v}$. Letting $N \rightarrow \infty$ yields the desired inequality.

\section{ACKNOWLEDGEMENT}

This article is dedicated to the memory of T. Lachand-Robert. This work was performed with the financial support of PAI Egide Brancusi. The authors would like to thank H. Brezis for pointing out reference [15]. Thanks also to H. Brezis and A. Farina for stimulating discussions and for their invaluable mathematical culture.

\section{References}

[1] A. Aftalion and W. Reichel, Existence of two boundary blow-up solutions for semilinear elliptic equations, J. Diff Eq. 141 (1997), 400-421.

[2] C. Bandle and M. Marcus, Large solutions of semilinear elliptic equations: existence, uniqueness and asymptotic behavior, J. Math. Anal. 58 (1992), 9-24.

[3] C. Bandle and M. Marcus, Asymptotic behaviour of solutions and their derivatives, for semilinear elliptic problems with blow-up on the boundary, Ann. Inst. Henri Poincaré 12 (1995), 155-171. 
[4] P. Ciarlet, Introduction à l'analyse numérique matricielle et à l'optimisation, Masson, 1988.

[5] Ph. Clément and G. Sweers, Existence and multiplicity results for a semilinear elliptic eigenvalue problem, Ann. della Scola Norm. Sup. di Pisa, (4), 14, n 1, (1987), 97-121.

[6] Ph. Clément and G. Sweers, Getting a solution between sub and supersolutions without monotone iteration, Rend. Istit. Mat. Univ. Trieste 19, n. 2, (1987), 189-194.

[7] Y. Du and Z. Guo, Liouville type results and eventual flatness of positive solutions for p-laplacian equations, Adv. in Diff. Eq. 7, n 12, (2002), 1479-1512.

[8] Y. Du and Z. Guo, Uniqueness and layer analysis for boundary blow-up solutions, J. Maths. Pures et Appl. 83 (2004), 739-763.

[9] B. Gidas, W-M. Ni and L. Nirenberg, Symmetry and related properties via the maximum principle, Comm. Math. Phy. 68 (1979), 209-243.

[10] J. Keller, On solutions to $\Delta u=f(u)$, Comm. Pure and Applied Math. 10 (1957), 503-510.

[11] S. Kishenassamy, Recent progress in boundary blow up, Elliptic and parabolic problems, Prog. in nonlinear diff. equations appl. 63, Birkhauser, Basel, (2005), 329-341.

[12] A. Lazer and P. McKenna, Asymptotic behaviour of solutions to boundary blow-up problems, Diff. and Int. Equations 7, n 4, (1994), 1001-1019.

[13] M. Marcus and L. Véron, Existence and uniqueness results for large solutions of general nonlinear elliptic equations, J. Evolution Equ. 3 (2003), 637-652.

[14] R. Osserman, On the inequality $\Delta u \geq f(u)$, Pacific J. Math. 7 (1957), 1641-1647.

[15] A. Poretta and L. Véron, Symmetry of large solutions of nonlinear elliptic equations in a ball, to appear. 